\title{
Evaluation of a developed IMB based-ELISA in diagnosis of urinary schistosomiasis in areas at risk in Upper Egypt
}

\section{Original Article}

\author{
Doreya M Mahmoud ${ }^{1}$, Ghada A Saad ${ }^{1}$, Ibrahim R Bayoumi², Zeinab M Abdel-Hady ${ }^{1}$, \\ Heba A Aminou ${ }^{1}$
}

Departments of Parasitology, Faculty of Medicine, Ain Shams University, Cairo ${ }^{1}$, and

Theodor Bilharz Research Institute, Giza², Egypt

\begin{abstract}
Background: Nanotechnology enables researchers to boost accuracy of the existing diagnostic techniques. Immunomagnetic beads (IMB) based-ELISA was utilized to diagnose several parasitic diseases; schistosomiasis mansoni and japonicum, toxoplasmosis and neurocysticercosis.

Objectives: The present work aims to develop and evaluate a novel nano-diagnostic assay using gold nanoparticles (AuNPs) in ELISA (IMB based-ELISA) for the diagnosis of urinary schistosomiasis.

Subjects and Methods: IMB based-ELISA assay was developed by preparation of polycolonal antibodies (pAbs) against Schistosoma soluble egg antigen (SEA). The developed novel assay was evaluated in urine samples of 290 schoolchildren collected from primary and preparatory schools in four villages in Beni-Suef governorate, Egypt. Urine samples were screened by chemical reagent strips (Combi 10) and examined by urine microscopy (UM). The conventional ELISA technique was used to evaluate the efficacy of IMB basedELISA using UM as the gold standard method for diagnosis of urinary schistosomiasis.

Results: The novel IMB based-ELISA assay succeeded to diagnose 50 out of 290 schoolchildren (17.2\%). In comparison with other methods, results showed that 39/290 (13.4\%) were positive by UM and $53 / 290(18.3 \%)$ by conventional ELISA. A sensitivity, specificity and diagnostic accuracy of the evaluated ELISA assay using UM as the gold standard method were $94.87 \%, 95.22 \%$ and $94.48 \%$ respectively. It was observed that Combi-10 gave sensitivity and specificity of $35.9 \%$ and $94.9 \%$ respectively for microhaematuria and proteinuria.

Conclusion: IMB-ELISA based on AuNPs provides a more rapid as well as sensitive detection of SEA in urine samples of patients with active schistosomiasis. Its high sensitivity and specificity ensure its application in field studies. Additionally, urinary schistosomiasis proved highly prevalent in schoolchildren living in Beni-Suef villages.
\end{abstract}

Keywords: Combi 10; conventional ELISA; Egypt, gold nanoparticles; IMB based ELISA; S. haematobium; urine microscopy.

Received: 27 November, 2020, Accepted: 17 March, 2021.

Corresponding Author: Heba A. Aminou, Tel.: +20 1001550924, E-mail: hebaaminou74@hotmail.com

Print ISSN: 1687-7942, Online ISSN: 2090-2646, Vol. 14, No. 1, April, 2021.

\section{INTRODUCTION}

Human schistosomiasis, also known as bilharzia, is a chronic parasitic disease, caused by blood flukes; Schistosoma ${ }^{[1]}$. It is an insidious yet highly destructive disease being the most common parasitic disease in the world, second only to malaria among tropical disease ${ }^{[2]}$ and third after intestinal helminthiasis in global parasitism ${ }^{[3]}$. Typically, the highest incidence of this disease is observed in schoolchildren ${ }^{[4]}$. Despite the availability of effective drugs, the annual death rate in sub-Sahara Africa alone is $\sim 200,000$ rendering it the most lethally neglected tropical disease (NTD) [2]. In 1997, the Egyptian National Schistosomiasis Control Program adopted a morbidity control strategy through mass treatment with praziquantel. By the end of 2010 , only 29 villages had prevalence more than $3 \%$ and none had more than $10 \%$. In 2018, Haggag et al. ${ }^{[5]}$ studied the prevalence of $S$. haematobium infection among schoolchildren in some Upper Egyptian governorates, where schistosomiasis haematobium is known to be prevalent. They found that the prevalence in Sohag, Beni-Suef and Minya governorates was $2.4 \%, 1.2 \%$ and $1.2 \%$, respectively. The great success in controlling schistosomiasis in Egypt is attained through the application of several control programs that comply the strategy recommended by the $\mathrm{WHO}^{[6]}$. Two forms are endemic in Egypt: intestinal and urinary schistosomiasis caused by $S$. mansoni, and $S$. haematobium, respectively. The latter is the only blood fluke that infects the urinary tract, predominating in south of Cairo along the Nile Valley ${ }^{[7]}$.

Schistosomiasis haematobium immunopathology is due to delayed hypersensitivity reaction that results in the development of granulomas around eggs followed by fibrosis leading to obstructive manifestations throughout the urinary tract. The stage of oviposition is represented by genitourinary disorders such as cystitis, dysuria with terminal hematuria, dull suprapubic pain, spermatorrhea and hemospermia. Spontaneous recovery is uncommon, 
and the condition may be complicated by bladder ulcers, calculi, polyps, fistulae, hydroureters, hydronephrosis or carcinomatous changes of the bladder ${ }^{[8]}$.

As schistosomiasis is a NTD of global importance, it is one of the priorities for global elimination programs. Implementation of accessible and inexpensive mass diagnostic screening tests with high sensitivity are therefore necessary. Over the past few decades, a variety of diagnostic techniques were developed, ranging from simple microscopic detection to sophisticated molecular approaches ${ }^{[9]}$.

Urine microscopy for detecting and counting $S$. haematobium eggs was commonly used as a standard diagnostic test of urogenital schistosomiasis because it is easy and cheap to perform in remote endemic regions. However, it lacks accuracy and sensitivity due to daily variations in the number of eggs excreted in individual patients especially in light infections ${ }^{[10]}$. Antibody detection methods are also limited particularly in areas where the disease is highly endemic due to their inability to distinguish between Schistosoma species, or differentiate between active or chronic infections. Also, errors may arise from false positive results attributed to cross reactions with antibodies from other helminth infections ${ }^{[11]}$. On the other hand, antigen detection assays represent a useful alternative diagnostic tool in two respects: first, the high sensitivity of the assays allow diagnosis of active infection; second, the antigen assays allow direct calculation of worm burden than quantitative parasitological techniques which would be extremely valuable for immuno-epidemiological studies $^{[12]}$. Detection of SEA in urine has several potential advantages: it is none invasive and its recognition correlates well with active infections ${ }^{[13]}$

Nanotechnology has the ability to use many different tools and technologies to boost the diagnostic accuracy of the existing techniques. This is due to the small size of NPs which makes their physical and chemical properties exceptional in comparison with their corresponding bulk materials ${ }^{[14]}$. In comparison to their corresponding materials, they are able to increase the sensitivity of different techniques as well as efficacy of several drugs. Additionally, AuNPs have been used in imaging assays due to their biocompatibility and high stability $^{[14]}$. In the era of nanodiagnosis, conjugation of antibodies or antigens (with their high sensitivity and specificity) with NPs (with their exceptional physical and chemical properties), increases the ability of different diagnostic techniques to detect target molecules ${ }^{[14]}$. Similarly, instead of using enzymes or fluorescent material as labels, the NPs bound to a biomolecule are used to capture the same target ${ }^{[15]}$. Besides, NPs can also be used as sole treatment or as a drug delivery system in treatment of many parasitic diseases such as giardiasis ${ }^{[16]}$, visceral leishmaniasis ${ }^{[17]}$ and fascioliasis ${ }^{[18]}$. In control and prevention, NPs are also used to vaccinate mice against toxoplasmosis ${ }^{[19]}$, and as adjuvant to ameliorate the immune response in schistosomiasis $^{[20]}$ and Chagas disease ${ }^{[21]}$.

In diagnosis of infectious and parasitic diseases, ELISA has been frequently used for antigen or antibody detection in clinical laboratories as it is specific, simple and of low cost ${ }^{[22]}$. However, ELISA has many disadvantages such as prolonged incubations and multiple washing steps. With the evolutionary advances in nanotechnology, IMB based-ELISA has been introduced in order to reduce the total time for analyses and improve the sensitivity ${ }^{[23]}$. Several publications utilized IMB based-ELISA to isolate nucleic acids, proteins and cells to be used in diagnosis of many parasitic infections as schistosomiasis mansoni ${ }^{[24]}$, schistosomiasis japonicum ${ }^{[25]}$, toxoplasmosis ${ }^{[26]}$, and neurocysticercosis ${ }^{[27]}$. In 2015, Hegazy et al. ${ }^{[26]}$ found that IMB based-ELISA has higher sensitivity (98\%), specificity (96.4\%), positive predictive value (96\%) and negative predictive value $(98.1 \%)$ than conventional ELISA when evaluated in the diagnosis of human toxoplasmosis using surface antigen (SAG1). In a study conducted by Méabed and Hassan ${ }^{[28]}$, IMB basedELISA proved to be a sensitive and specific method for diagnosis of $S$. mansoni in patients with low-intensity infections.

In the present work, we developed IMB based-ELISA, and applied it to diagnose urinary schistosomiasis for detection of SEA of $S$. haematobium in urine samples. Our study aims to evaluate the developed novel assay by comparing its diagnostic accuracy with conventional ELISA using UM as reference gold standard test Assessment of its application in field studies will be useful for screening urinary schistosomiasis among schoolchildren in areas at high risk in Upper Egypt.

\section{SUBJECTS AND METHODS}

This descriptive analytical study was conducted in Parasitology Department, Faculty of Medicine, Ain Shams University, Cairo, and Research Laboratory of Theodor Bilharz Research Institute (TBRI), Giza, Egypt during the period from October to November 2018.

Study design: Urine samples were collected from 290 schoolchildren from primary and preparatory schools in four villages in Beni-Suef governorate. Collected urine samples were subjected to microscopy for egg detection at Medical Parasitology Department, Faculty of Medicine, Ain Shams University. According to UM results (presence or absence of E. vermicularis eggs), study population was divided into three groups. Urine samples in each group were individually examined by IMB based-ELISA and conventional ELISA for SEA detection at TBRI Research Laboratory.

Study population and urine sample collection: The novel IMB based-ELISA assay was evaluated 
for 290 participants from primary and preparatory schools in four villages in Beni-Suef governorate (El Shantour, Ezbet El Shantour, El Sheikh Khattab and Kaftan villages) where urinary schistosomiasis is highly prevalent. By age, 250 (86.2\%) were 8-12 years, and $40(13.8 \%)$ were $13-15$ years old. By gender, 98 (33.8\%) were girls, 192 (66.2\%) were boys. At first, a structured questionnaire was filled out for each participant. It included data on their sociodemography, and clinical history as well as factors that influence endemicity of urinary schistosomiasis. Sociodemographic data included, age, sex, highest level of education, and residence. Clinical history comprised signs and symptoms of urinary schistosomiasis as abdominal pain, urine colour, haematuria, and dysuria. Factors that influence the prevalence and endemicity of urinary schistosomiasis are supply and source of drinking water, site of bathing and number of water bodies used in the locality, and participation in agricultural activities.

Urine samples were collected from each participant in sterile, wide mouthed, screw capped plastic container with the instructions to deposit terminal drops of urine. The containers were labelled with student name. Immediately, samples were tested for micro-haematuria and proteinuria using Combi 10 (Medi-Test, Germany) for rapid determination of blood and protein in urine. Each urine sample was divided into two falcons. The first was subjected to microscopic examination of urine sediment after centrifugation for $5 \mathrm{~min}$ at $2000 \mathrm{~g}$ for detection of $S$. haematobium eggs, in the Department of Medical Parasitology, Faculty of Medicine, Ain Shams University. The second one was properly corked and transported in cool boxes to the Research Laboratory of the TBRI for nano-immunoassay after addition of few drops of thiomersal compound as a preservative and conserved by refrigeration $\left(4^{\circ} \mathrm{C}\right)$.

\section{Development of anti-S. haematobium pAbs}

Animals: Two New Zealand white male rabbits, weighing approximately $1.5 \mathrm{~kg}$, purchased from Rabbit Research Unit, at Faculty of Agriculture, Cairo University were used for the production of anti-S. haematobium polyclonal antibodies (pAbs). They were examined and confirmed to be free from parasitic infections. The rabbits were housed in the animal house at TBRI for 4 weeks (experiment duration) under standard laboratory care.

Antigen: S. haematobium SEA used in this study was purchased from TBRI. This antigen was used to immunize rabbits for pAbs production.

Preparation of pAbs against SEA: Just before immunization, rabbits' sera were evaluated using ELISA for anti-schistosomal antibodies as well as crossreactivity with other parasitic infections. Each rabbit received $1 \mathrm{mg}$ of $S$. haematobium SEA intramuscularly, and same volume of complete Freund's adjuvant (CFA) (Pierce, Rockford, IL, USA). Booster doses [0.5 mg of SEA antigen with an equal volume of incomplete Freund's adjuvant (IFA) (Sigma, Germany)] were given at weeks 2, 3 and 4 after the first one ${ }^{[29]}$. After the last dose by one week, rabbit's serum was obtained and pAb fraction was purified using 50\% ammonium sulphate precipitation method ${ }^{[30]}$. Further purification of pAb was performed by $7 \%$ caprilic acid method ${ }^{[31]}$ and finally with gel-filtration ${ }^{[32]}$. Protein content was measured after each purification using Bradford method $^{[33]}$. Partially purified pAb was further adsorbed with fetal calf serum to remove non-specific binding with bovine antigen.

Performance of IMB based-ELISA: Microtitration plates were coated using $100 \mu \mathrm{l}$ anti-S. haematobium pAbs in each well, coupled with $10 \mu \mathrm{g} / \mathrm{ml}$ AuNPs magnetic microbeads (Sigma Aldrich, Germany) in carbonate buffer $0.06 \mathrm{M}$ (pH 9.6). Plates were incubated overnight at room temperature, washed three times with $0.1 \mathrm{M} \mathrm{PBS} / \mathrm{T}$ ( $\mathrm{pH} 7.4$ ), and the remaining wells were blocked with $200 \mu \mathrm{l} 2.5 \%$ fetal calf serum/PBS/ Tween followed by incubation for $2 \mathrm{~h}$ at $37^{\circ} \mathrm{C}$ and washing 3 times with PBS/T. Undiluted urine samples $(100 \mu \mathrm{l})$ were added into the wells in duplicate and incubated for $2 \mathrm{~h}$ at $37^{\circ} \mathrm{C}$. The wells were then washed 5 times with washing buffer. Finally, $100 \mu \mathrm{l} /$ well of $1 / 3000$ peroxidase-conjugated polyclonal antibodies were added and incubated for $2 \mathrm{~h}$ at room temperature, and then washed 5 times as before. The reaction was visualized by adding $100 \mu \mathrm{l} /$ well of 0 -phenylene diamine (OPD) substrate solution for $30 \mathrm{~min}$ at room temperature, in the dark. The reaction was stopped by the addition of $50 \mu \mathrm{l} /$ well of $8 \mathrm{~N} \mathrm{H2SO} 4$ and plates were finally read at $492 \mathrm{~nm}$ using ELISA microplate reader (Bio Rad, Richmond, Ca, USA).

Performance of conventional ELISA ${ }^{[34]}$ : It was performed after several trials for optimization. Microtitration plates (Dynatech) were coated with 100 $\mu \mathrm{l} /$ well of $10 \mu \mathrm{g} / \mathrm{ml}$ purified pAbs in $0.06 \mathrm{M}$ carbonate buffer (pH 9.6), and left overnight at room temperature. Wells were blocked by $200 \mathrm{ul} 2.5 \%$ fetal calf serum/ PBS/Tween in each well, for $2 \mathrm{~h}$ at $37^{\circ} \mathrm{C}$. The wells were then washed 3 times with washing buffer 0.01 M PBS 0.05\% Tween, pH 7.2). Undiluted urine samples were pipetted (100 $\mu \mathrm{l} /$ well) and incubated for $2 \mathrm{~h}$ at $37^{\circ} \mathrm{C}$. Plates were washed 5 times with washing buffer. Finally, $100 \mu \mathrm{l} /$ well of peroxidase conjugated pAbs $(25 \mu \mathrm{g} / \mathrm{ml})$ were added and incubated for $2 \mathrm{~h}$ at room temperature, followed by 5 times washing as before. The reaction was then visualized as previous.

Statistical analysis: Recorded data were analyzed using the Statistical Package for Social Sciences, version 20.0 (SPSS Inc., Chicago, Illinois, USA). Chi-square $\left(X^{2}\right)$ test of significance was used in order to compare proportions between qualitative parameters. GraphPad Prism 8.0 was used for presenting data like scatter plots. 
Sensitivity, specificity, positive and negative predictive values (PPV and NPV), and diagnostic accuracy were calculated as parameters to evaluate IMB based ELISA. Statistical significance is considered when $P$ value $<0.05$.

Ethical consideration: This study was reviewed and approved by the Ain Shams University Ethical Committee that complies with the 1964 Helsinki declaration. Methods including samples election were performed in compliance with applicable guidelines and regulations. Animal experiments were conducted in accordance with the Guide for Care and Use of Laboratory Animals of the National Institutes of Health (NIH) and were approved by the Institutional Review Board of TBRI. All methods involving animals; immunization, fusion procedure, and large-scale production of pAbs were carried out in accordance with relevant guidelines and regulations. Ethical approval was obtained from the authorized Ministry of Health and Population. Oral consent from guardians and children was obtained before enrolment. The purpose and procedures of the study were explained to school officials and local health authorities. The study topic was explained to the participants before obtaining the sample. Infected schoolchildren were informed to start treatment.

\section{RESULTS}

According to UM results, study participants were divided into 3 groups, 39 were positive for $S$. haematobium (group A); 14 were positive for $E$. vermicularis but negative for $S$. haematobium (group B); and 237 schoolchildren served as a control group with negative parasitic infection (group C) (Table 1).
Table 1. Study groups as determined by direct microscopic examination of urine sediments $(n=290)$.

\begin{tabular}{llcc}
\hline \hline Group & \multicolumn{1}{c}{ Infection status } & No. & \% \\
\hline A & Positive for S. haematobium only & 39 & 13.45 \\
B & Positive for E. vermicularis only & 14 & 4.83 \\
C & Negative for S. haematobium and other parasites & 237 & 81.72 \\
\hline Total & & $\mathbf{2 9 0}$ & $\mathbf{1 0 0}$ \\
\hline \hline
\end{tabular}

The novel IMB based-ELISA assay succeeded to diagnose 50 cases out of 290 schoolchildren (17.2\%). In comparison with other methods, results showed that $39 / 290$ (13.4\%) were positive by UM and $53 / 290$ (18.3\%) by conventional ELISA. A sensitivity, specificity and diagnostic accuracy of IBM based-ELISA using UM as the gold standard method were $94.87 \%, 95.22 \%$ and $94.48 \%$ respectively, while those of conventional ELISA were $87.2 \%, 92.4 \%$ and $91.72 \%$. There was statistically significant difference between UM and both ELISA assays $(P<0.00001)$ (Table 2$)$. It was observed that optical density (OD) of IBM based-ELISA was 0.6 for the positive cases (Figure 1), while that of conventional ELISA was 0.4 (Figure 2).

Table (3) demonstrated the comparison between microhaematuria, proteinuria and the true disease status as determined by UM. Microhaematuria was detected in 62 students; 18 of them were positive for $S$. haematobium eggs while 44 were negative. The remaining samples with negative microhaematuria (No.=228) showed that 21 had $S$. haematobium eggs in their urine and 207 were devoid of eggs. Sensitivity, specificity and diagnostic accuracy of microhaematuria, were $29.51 \%, 90.83 \%$ and $77.93 \%$, respectively. Proteinuria was detected in 158 samples; among which 29 were positive for $S$. haematobium eggs in urine, and 129 were negative. In the remaining samples with negative proteinuria (No.=132), 10 had S. haematobium

Table 2. Evaluation of IMB based-ELISA and conventional ELISA in comparison to UM in 290 study population.

\begin{tabular}{|c|c|c|c|c|c|c|}
\hline & \multicolumn{4}{|c|}{ UM } & \multicolumn{2}{|c|}{ Statistical analysis } \\
\hline & Group (A) & Group (B) & Group (C) & Total & $X^{2}$ & $P$ value \\
\hline \multicolumn{7}{|l|}{ IMB based-ELISA } \\
\hline Positive [No. (\%)] & 37 (94.9) & $0(0)$ & $13(5.5)$ & 50 & \multirow{8}{*}{190.95} & \multirow{8}{*}{$<0.00001$} \\
\hline Negative [No. (\%)] & $2(5.1)$ & $14(100)$ & $224(94.5)$ & 240 & & \\
\hline Total & 39 & 14 & 237 & 290 & & \\
\hline Sensitivity\% & 94.87 & & & & & \\
\hline Specificity $\%$ & 95.22 & & & & & \\
\hline PPV\% & 73.47 & & & & & \\
\hline NPV\% & 98.76 & & & & & \\
\hline Diagnostic accuracy\% & 94.48 & & & & & \\
\hline \multicolumn{7}{|l|}{ Conventional ELISA } \\
\hline Positive [No. (\%)] & $34(87.8)$ & $0(0)$ & $19(8.0)$ & 53 & \multirow{3}{*}{143.8} & \multirow{3}{*}{$<0.00001$} \\
\hline Negative [No. (\%)] & $5(12.2)$ & $14(100)$ & $218(92.0)$ & 237 & & \\
\hline Total & 39 & 14 & 237 & 290 & & \\
\hline Sensitivity\% & 87.2 & & & & & \\
\hline Specificity\% & 92.4 & & & & & \\
\hline PPV\% & 64.15 & & & & & \\
\hline NPV\% & 97.89 & & & & & \\
\hline Diagnostic accuracy\% & 91.72 & & & & & \\
\hline
\end{tabular}




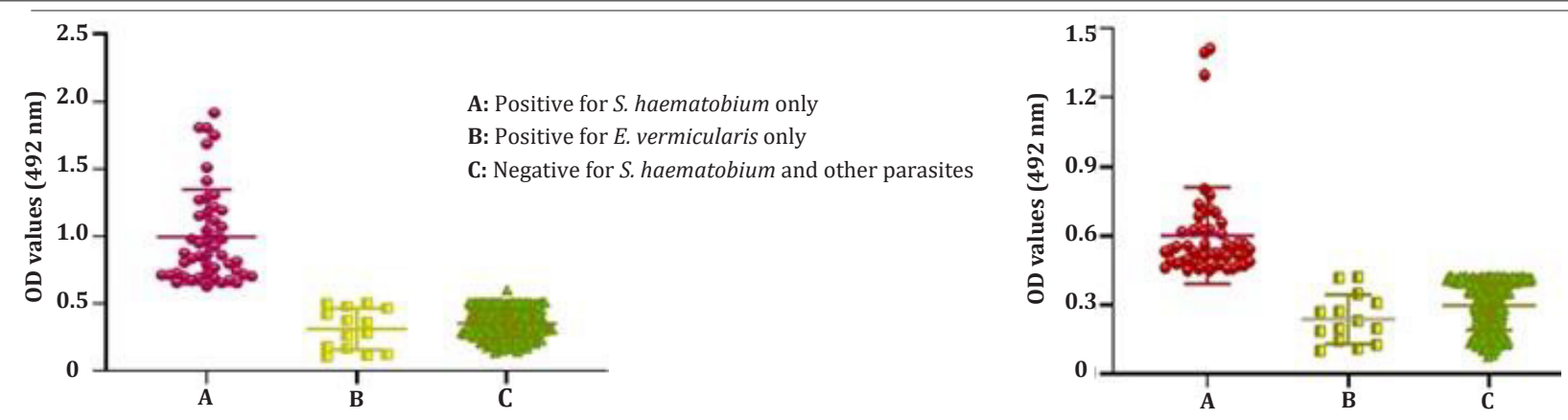

Fig. 1. Scatter plot curve showing the diagnostic performance of IMB based-ELISA.

eggs in urine and 122 were devoid of eggs. Sensitivity, specificity and diagnostic accuracy of proteinuria were $18.35 \%, 92.42 \%$ and $52.07 \%$, respectively. Both microhaematuria and proteinuria were detected in 39 students; among them 14 were positive for $S$. haematobium eggs while 25 were negative. The
Fig. 2. Scatter plot curve showing the diagnostic performance of conventional ELISA.

remaining samples with negative microhaematuria and proteinuria (No.=109) showed that 6 had $S$. haematobium eggs in their urine and 103 were devoid of eggs. Sensitivity, specificity and diagnostic accuracy of both indicators were $35.9 \%, 94.5 \%$ and $79.05 \%$, respectively.

Table 3. Sensitivity, specificity, and diagnostic accuracy of Combi 10 according to UM results.

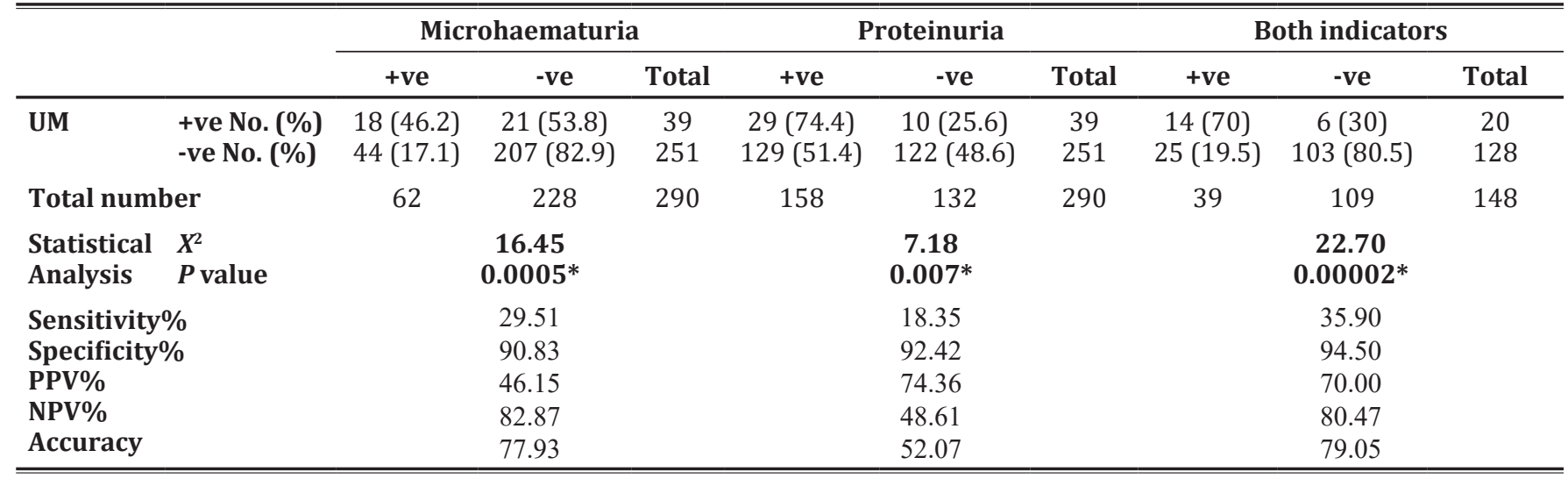

No.: Number; +ve: Positive; -ve: Negative; PPV: Positive predictive value; NPV: Negative predictive value; *: Significant $(P<0.05)$.

Table (4) presents results of UM in four different schools revealing the number of boys (28/192) and girls (11/98) students with positive $S$. haematobium ova in urine. According to age group, 35 school children out of 250 had $S$. haematobium ova in their urine in the age group 8-12 years, while concerning age group 1315 years, only 4 out of 40 students were positive. The results were insignificant $(P>0.05)$.

Table 4. Urinary schistosomiasis according to schools, sex, and age groups.

\begin{tabular}{|c|c|c|c|c|c|}
\hline \multirow[b]{2}{*}{ Variable } & \multirow[b]{2}{*}{ No. } & \multicolumn{2}{|c|}{ UM } & \multicolumn{2}{|c|}{ Statistical analysis } \\
\hline & & $\begin{array}{l}\text { Positive } \\
\text { No. (\%) } \\
\end{array}$ & $\begin{array}{c}\text { Negative } \\
\text { No. (\%) }\end{array}$ & $X^{2}$ & $P$ value \\
\hline \multicolumn{6}{|l|}{ School } \\
\hline Mahmoud Fahmy & 60 & $8(13.3)$ & $52(86.7)$ & \multirow{5}{*}{5.29} & \multirow{5}{*}{$0.152^{\mathrm{NS}}$} \\
\hline Ezbet Al -Shantour & 60 & $3(5 \%)$ & $57(95)$ & & \\
\hline Sheikh Khattab & 60 & $11(18.3)$ & 49 (81.7) & & \\
\hline Kaftan & 110 & $17(15.5)$ & $93(84.5)$ & & \\
\hline Total & 290 & $39(13.4)$ & $251(86.6)$ & & \\
\hline \multicolumn{6}{|l|}{ Sex } \\
\hline Male & 192 & $28(14.6)$ & $164(85.4)$ & \multirow{2}{*}{0.629} & \multirow{2}{*}{$0.428^{N S}$} \\
\hline Female & 98 & $11(11.2)$ & $87(88.8)$ & & \\
\hline \multicolumn{6}{|l|}{ Age } \\
\hline 8-12 & 250 & $35(14)$ & $215(86)$ & \multirow{2}{*}{0.474} & \multirow{2}{*}{$0.152^{\mathrm{NS}}$} \\
\hline $13-15$ & 40 & $4(10)$ & $36(90)$ & & \\
\hline
\end{tabular}




\section{DISCUSSION}

Schistosomiasis is one of the most common occupational diseases, acquired by contact with freshwater during farming, bathing and washing. It has a great impact on socio-economic and public health ${ }^{[35]}$. In Egypt, urinary schistosomiasis still represents a serious health problem that needs concentration. Its complications add more load on the national control programs and economy. In Egypt, a decrease in the prevalence of human schistosomiasis has been recorded due to control programs over the last 10 years, though the disease is still endemic in many foci ${ }^{[36]}$. In some cases, as in the acute phase of the disease or in patients with light infection, the parasitological diagnostic methods have low sensitivity and may lead to inaccurate evaluation of prevalence and intensity of infection. To overcome this defect, immunological tests used for diagnosing schistosomiasis were introduced $^{[15]}$. It was observed that antigen detection assays may contribute in early diagnosis more than antibody tests, as production of detectable levels of immunoglobulin needs time ${ }^{[37]}$.

Detection of schistosomal antigens, whether schistosomular, adult worms, or eggs, in different body fluids is now a validated and highly effective diagnostic method. Adult worm microsomal and circulating antigens are the most commonly used ${ }^{[38]}$. Circulating anodic antigen (CAA) and circulating cathodic antigen (CCA) are antigens excreted by adult Schistosoma worm into host blood stream ${ }^{[39]}$. Since they are released from living worms, the concentrations of these antigens correlate with the worm burden and their presence in blood indicates active infection. Circulating antigen detection provides sensitive and rapid diagnosis of schistosomiasis with promising results ${ }^{[40]}$. However, limitations of their use, referring to low sensitivity especially with very low worm burdens, was reported ${ }^{[41]}$. When used on urine samples, CAA and CCA also yielded low sensitivity compared with serum due to their lower concentrations in urine than in blood ${ }^{[42]}$. Detection of SEA in urine has many advantages; it is non-invasive, its presence indicates active infections and can provide information on anti-fecundity immunity. In addition, SEA detection has the potential for both diagnosis and morbidity assessment ${ }^{[42]}$. Huang et al. ${ }^{[43]}$ stated that use of SEA in ELISA is sensitive, can reduce cross-reactivity with samples from cases infected with other parasites and also improve its value in estimating therapeutic efficacy.

The present research aims to develop a novel nano-diagnostic assay using AuNPs (in an IMB basedELISA), that utilizes a larger surface area, for achieving higher sensitivity for detection of urinary SEA in urine of human schistosomiasis haematobium. The test was evaluated by comparing its diagnostic accuracy with conventional ELISA using UM as reference gold standard test. Assessment of its application in field studies will be useful for screening urinary schistosomiasis among schoolchildren in areas with high risk in Upper Egypt. Moreover, indirect screening was done by Combi 10 for micro-haematuria and proteinuria. Epidemiologic studies indicate that 10 to 15 year old children typically carry the highest rates of acute schistosomiasis and associated inflammation. The more lethal outcomes of infection with organ fibrosis are more common among adult age group ${ }^{[44]}$.

On detection of $S$. haematobium SEA in urine samples by IMB based-ELISA using AuNPs conjugated with antischistosomal polyclonal antibody (pAb), 2/39 (5.1\%) $S$. haematobium infected samples showed false negative results giving a sensitivity of $94.9 \%$ while $13 / 237$ $(5.5 \%)$ of the negative group were positive for SEA with a specificity of $95.2 \%$. No cross reaction was obtained with the other parasites-infected group (Table 2, Fig. 1). Concerning detection of SEA with conventional ELISA, 4 out of $39 S$. haematobium infected samples showed false negative results giving a sensitivity of $87.2 \%$ while 19 out of 237 of the negative group were positive for SEA giving a specificity of $92.4 \%$ (Table 2, Fig. 2). These false negative results were found in patients with low numbers of eggs $/ 10 \mathrm{~mL}$ urine and this may be attributed to the release of small undetectable amounts of antigen by intact ova of $S$. haematobium. Another possibility is that antigen released by the parasite forms immune complexes with circulating antibodies ${ }^{[45]}$. No cross reaction was obtained with the other parasitesinfected group. Similarly, Mahfouz et al. ${ }^{[46]}$ detected SEA in urine samples of $S$. haematobium infected patients, with a sensitivity of $90.66 \%$ and a specificity of $93.75 \%$.

Consequently, there was a general increase in the prevalence rate among participants when evaluated by IMB based ELISA. So, application of the nanomagnetic beads significantly increases the sensitivity and the specificity of the technique towards higher detection of the antigen in cases with light infections. Aly et al. ${ }^{[15]}$ also developed a novel IMB based ELISA using IgG to detect $S$. haematobium circulating microsomal fraction antigen. They reported sensitivity and specificity of $96.5 \%$ and $96.3 \%$ respectively, compared to $88.2 \%$ and 87.3\% obtained by conventional ELISA.

Using UM, it was found that 39 of the schoolchildren were positive with a detection rate of $13.4 \%$ of urinary schistosomiasis (Table 1). This was similar to previous studies performed among pre-school children in Egypt $(14.5 \%)^{[47]}$ and infants in Ghana ${ }^{[48]}$.

Statistical analysis showed no significant difference between males and females $(P>0.05)$ (Table 4) indicating that, both males and females are equally at risk of being infected. This finding is not consistent with the observations of Nanvya et al. ${ }^{[49]}$ in Nigeria who reported that females were less likely to be infected, but it is consistent with the reports of Akinboye et al. ${ }^{[50]}$ in Ibadan who found that both sexes are at equal risk. 
Our study showed similar high levels of infection in 2 age groups: 8-12 and 13-15 years old with an insignificant statistical difference between them $(\mathrm{P}>0.05)$ (Table 4); however, indicating that both age groups are at equal risk of infection. In agreement Abdel-Wahab et al. ${ }^{[51]}$ stated that the peak prevalence of $S$. haematobium infections in Egypt occur between the ages of 9-15 years old.

Statistical analysis showed no significant difference in the prevailing rates between studied schools $(P>$ $0.05)$. This can be explained by the fact that, people living in these areas rely on canals and ponds (in which schistosome parasites together with their intermediate hosts live) to get water. Continuous exposure to the parasite will lead to repeated infections.

In the present study, no visible haematuria was recorded by the children in the applied questionnaire. By chemical reagent strips (Combi 10), micro-haematuria and proteinuria showed a significant association with $S$. haematobium infection. The combined microhaematuria and micro-proteinuria was significantly assessed with $35.9 \%$ sensitivity, $94.5 \%$ specificity, PPV of $70 \%$, NPV of $80.5 \%$ and accuracy of $79.1 \%$ (Table $3)$. The high sensitivity values reflect the usefulness of these diagnostic indices as morbidity indicators of $S$. haematobium in an endemic area. These results are closely related to the report of Yameny ${ }^{[52]}$, indicating that when both micro-haematuria and proteinuria were considered, sensitivity and specificity increased to $45.2 \%$ and $85.9 \%$ respectively.

However, micro-haematuria and proteinuria are nonspecific signs that can be associated with other diseases such as urogenital infection, malignancy, immune system disorders and trauma. Consequently, urine reagent strips for combined proteinuria and micro-haematuria might have a diagnostic value only in endemic areas where microscopy is not available ${ }^{[52]}$.

In conclusion, combination of both clinical and epidemiological data in addition to sensitive diagnostic tools is essential for diagnosis. The present study as with other studies revealed that, IMB-ELISA based on AuNPs provides more rapid and sensitive detection for SEA in urine samples of patients with active schistosomiasis. Simplicity and fast detection (10 $\mathrm{min})$ are its main advantages. Moreover, its high sensitivity and specificity ensures its application with greater precision and rapid detection.

Authors contribution: Mahmoud DM designed the plan of work and revised the manuscript. Saad GA shared in designing the plan of work, analyzing the data, and revising the manuscript. Bayoumi IR shared in performing the practical part and analyzing the data. Abdel-Hady ZM performed the practical part, analyzed the data, and shared in writing the manuscript. Aminou HA shared in designing the plan of work, analyzing the data, and revising the manuscript.
Conflicts of interest: We wish to confirm that there are no known conflicts of interest associated with this publication. We confirm that the manuscript has been read and approved by all named authors and ensure that there are no other persons who satisfied the criteria for authorship but are not listed. We further confirm that the order of authors listed in the manuscript has been approved by all authors.

Financial support: There has been no financial support for this work that could have influenced its outcome.

\section{REFERENCES}

1. Anon. Schistosomiasis. WHO Fact Sheet. WHO Media Centre. Retrieved 12 December 2017.

2. Karunamoorthi K, Almalki MJ, Ghailan KY. Schistosomiasis: A neglected tropical disease of poverty: A call for intersectoral mitigation strategies for better health. J Health Res Rev 2018; 5(1):1.

3. Okwori AJ, Sidi M, Ngwai YB, Obiekezie SO, Makut MD, Chollom SC, et al. Prevalence of schistosomiasis among primary school children in Gadabuke District, Toto LGA, North Central Nigeria. Br Microbiol Res J 2014; 4: 255-261.

4. Stothard JR, Sousa-Figueiredo JC, Betson M, Bustinduy A, Reinhard-Rupp J. Schistosomiasis in African infants, and preschool children: let them now be treated! Trends Parasitol 2013; 29(4):197205.

5. Haggag AA, Rabiee A, Abd Elaziz KM, Gabrielli AF, Abdelhai R, Hashish A, et al. Elimination of schistosomiasis haematobium as a public health problem in five governorates in Upper Egypt. Acta Trop 2018; 188:9-15.

6. Barakat RMR. Epidemiology of schistosomiasis in Egypt: Travel through time: Review. J Adv Res 2013; 4(5):425-432.

7. Antoni S, Ferlay J, Soerjomataram I, Znaor A, Jemal A, Bray F. Bladder cancer incidence and mortality: a global overview and recent trends. Eur Urol 2017; 71(1):96-108.

8. Wu GY, Halim MH. Schistosomiasis: progress and problems. World J Gastroenterol 2000; 6(1):12.

9. Weerakoon KG, Gobert GN, Cai P, McManus DP. Advances in the diagnosis of human schistosomiasis. Clin Microbiol Rev 2015; 28(4):939-967.

10. Song HB, Kim J, Jin Y, Lee JS, Jeoung HG, Lee YH, et al. Comparison of ELISA and urine microscopy for diagnosis of Schistosoma haematobium infection. J Korean Med Sci 2018; 33(33):e238.

11. Ajibola O, Gulumbe BH, Eze AA, Obishakin E. Tools for detection of schistosomiasis in resource limited settings. Med Sci 2018; 6(2):39.

12. Ibrahim RB, Mohamed SH, Demerdash ZA, Diab TM, Maher K, Safwat W, et al. Anti-S. mansoni mAbbased latex agglutination: a reliable field applicable immunodiagnostic test for screening of active human schistosomiasis. Am J Sci 2010; 6:19-27. 
13. Kahama AI, Nibbeling HA, Van Zeyl RJ, Vennervald BJ, Ouma JH, Deelder AM. Detection and quantification of soluble egg antigen in urine of Schistosoma haematobium-infected children from Kenya. Am J Trop Med Hyg 1998; 59(5):769-774.

14. Odundo J, Noah N, Andala D, Kiragu J, Masika. Development of an electrochemical nano-biosensor for rapid and sensitive diagnosis of bilharzia in Kenya. S Afr J Chem 2018; 71(1):127-134.

15. Aly I, Zalat R, El Aswad BW, Moharm IM, Masoud BM, Diab T. Novel nanomagnetic beads based-latex agglutination assay for rapid diagnosis of human schistosomiasis haematobium. Int J Med Health Biomed Bioeng Pharm Eng 2013; 7(12):642-647.

16. Said DE, Elsamad LM, Gohar YM. Validity of silver, chitosan, and curcumin nanoparticles as anti-Giardia agents. Parasitol Res 2012; 111:545-554.

17. Das S, Roy P, Mondal S, Bera T, Mukherjee A. One pot synthesis of gold nanoparticles and application in chemotherapy of wild and resistant type visceral leishmaniasis. Colloids Surf B Biointerfaces 2013; 107:27-34.

18. Gherbawy YA, Shalaby IM, El-Sadek MS, Elhariry HM, Abdelilah BA. The anti-fasciolasis properties of silver nanoparticles produced by Trichoderma harzianum and their improvement of the anti-fasciolasis drug triclabendazole. Int J Mol Sci 2013; 14:21887-21898.

19. El Bissati K, Zhou Y, Dasgupta D, Cobb D, Dubey JP, Burkhard P et al. Effectiveness of a novel immunogenic nanoparticle platform for Toxoplasma peptide vaccine in HLA transgenic mice. Vaccine 2014; 32:3243-3248.

20. Wu J, Peng Y, Liu X, Li W, Tang S. Evaluation of Wondfo rapid diagnostic kit (Pf-HRP2/PAN$\mathrm{pLDH}$ ) for diagnosis of malaria by using nano-gold immunochromatographic assay. Acta Parasitol 2014; 59:267-271.

21. Higa LH, Corral RS, Morilla MJ, Romero EL, Petray PB. Archaeosomes display immunoadjuvant potential for a vaccine against Chagas disease. Hum Vaccin Immunother 2013; 9:409-412.

22. Sakamoto S, Putalun W, Vimolmangkang S, Phoolcharoen W, Shoyama Y, Tanaka H et al. ELISA for the quantitative/qualitative analysis of plant secondary metabolites. J Nat Med 2018; 72(1):32-42.

23. Wu L, Li G, Xu X, Zhu L, huang R, Chen X. Application of nano-ELISA in food analysis: Recent advances and challenges. Trends Anal Chem 2019; 113:140-156.

24. Teixeira, CF, Neuhauss, E, Ben, R, Romanzini J, GraeffTeixeira C. Detection of $S$. mansoni eggs in feces through their interaction with paramagnetic beads in a magnetic field. PLoSNegl Trop Dis 2007; 1(2):e73

25. Yu Q, Yang H, Guan F, Feng Y, Yang X, Zhu Y. Detection of IgG in sera of patients with schistosomiasis japonica by developing magnetic affinity enzyme-linked immunoassay based on recombinant 14-3-3 protein. Trans R Soc Trop Med Hyg 2014; 108(1):37-41.

26. Hegazy S, Farid A, Rabae I, El-Amir A. Novel IMB-ELISA assay for rapid diagnosis of human toxoplasmosis using SAG1 antigen. Jpn J Infect Dis 2015; 68(6):474480 .
27. Hernández-González A, Noh J, Perteguer MJ, Gárate T, Handali S. Comparison of T24H-his, GST-T24H and GST-Ts8B2 recombinant antigens in western blot, ELISA, and multiplex bead-based assay for diagnosis of neurocysticercosis. Parasit Vectors 2017; 10(1):2327.

28. Méabed EMH, Hassan EA. Immuno-magnetic beads ELISA for diagnosis of $S$. mansoni infection. J Egypt Soc Parasitol 2019; 49(1):51-59.

29. Fagbemi BO, Aderibigbe OA, Guobadia EE. The use of monoclonal antibody for the immune diagnosis of Fasciola gigantica infection in cattle. Vet Parasitol 1997; 69(3-4):231-240.

30. Odunuge 00, Shazhko A. Ammonium sulfate precipitation combined with liquid chromatography is sufficient for purification of bovine serum albumin that is suitable for most routine laboratory applications. Biochem Comp 2013; 1(1): 3.

31. Sheehan D, FitzGerald R. Ion-exchange chromatography. Methods Mol Biol 1996; 59:145150.

32. McKinney MM, Parkinson A. A simple, nonchromatographic procedure to purify immunoglobulins from serum and ascites fluid. J Immunol Meth 1987; 96(2):271-278.

33. Bradford MA. Rapid and sensitive method for the quantitation of microgram quantities of protein utilizing the principle of protein-dye binding. Ann Biochem 1976; 72:245-254.

34. Engvall E, Perlmann P. Enzyme-linked immunosorbent assay (ELISA) quantitative assay of immunoglobulin G. Immunochemistry 1971; 8(9):871-874.

35. Ekpo UF, Laja-Deile A, Oluwole AS, Sam-Wobo SO, Mfiana CF. Urinary schistosomiasis among preschool children in a rural community near Abeokuta, Nigeria. Parasit Vectors 2010; 3:58-62.

36. Aly IR. Efficacy of iron oxide nanoparticles in diagnosis of schistosomiasis. Al-Azhar Intern Med J 2020; 1(1): 219-224.

37. Moustafa NE, Hegab MH, Hassan MM. Role of ELISA in early detection of Fasciola copro-antigens in experimentally infected animals. J Egypt Soc Parasitol 1998; 28(2):379.

38. Al-Sherbiny MM, Osman AM, Hancock K, Deelder AM, Tsang VC. Application of immunodiagnostic assays: Detection of antibodies and circulating antigens in human schistosomiasis and correlation with clinical findings. Am J Trop Med Hyg 1999; 60(6):960-956.

39. Corstjens PL, De Dood CJ, Kornelis D, Tjon Kon Fat EM, Wilson RA, Kariuki TM et al. Tools for diagnosis, monitoring and screening of Schistosoma infections utilizing lateral-flow based assays and up-converting phosphor labels. Parasitology 2014; 141:1841-1855.

40. Shane HL, Verani JR, Abudho B, Montgomery SP, Blackstock AJ, Mwinzi PN. Evaluation of urine CCA assays for detection of $S$. mansoni infection in Western Kenya. PLoS Negl Trop Dis 2011; 5(1):e951.

41. Bustinduy AL, Waterhouse D, de Sousa-Figueiredo JC, Roberts SA, Atuhaire A, Van Dam GJ et al. Population pharmacokinetics and pharmacodynamics of 
praziquantel in Ugandan children with intestinal schistosomiasis: higher dosages are required for maximal efficacy. mBio 2016; 7(4):e00227-16.

42. Corstjens PL, Nyakundi RK, de Dood CJ, Kariuki TM, Ochola EA, Karanja DM, et al. Improved sensitivity of the urine CAA lateral-flow assay for diagnosing active Schistosoma infections by using larger sample volumes. Parasit Vectors 2015; 8:241.

43. Huang Y, Yi X, Zeng X, Zhang R, Yuan S. Study on diagnosis of schistosomiasis by ELISA using periodatetreated soluble egg antigen. Chinese J Parasitol Parasit Dis 2003; 21(4):238-241.

44. Van der Werf MJ, de Vlas SJ, Brooker S, Looman CWN, Nagelkerke NJD, Habbema JDF, Engels D. Quantification of clinical morbidity associated with schistosome infection in sub-Saharan Africa. Acta Trop 2003; 86:125-139.

45. Nash TE. Immune complex size determines the clearance rate of a circulating antigen in schistosomeinfected mice. Am J Trop Med Hyg 1984; 33(4):621626.

46. Mahfouz A, Mahana N, Rabee I, El-Amir A. Evaluation of different immunological techniques for diagnosis of schistosomiasis haematobium in Egypt. Life Sci J 2011; 8(4): 858-67.
47. Nour NM, Fayed MA, Hussein SM, Shaheen HI, AbdelRaheam RS. Prevalence of morbidity of schistosomiasis among preschool children in Bani-Suef governorate. J Egypt Soc Parasitol 1990; 20(2):793-803.

48. Bosompem KM, Bentum IA, Otchere J, Anyan WK, Brown CA, Osada Y, et al. Infant schistosomiasis in Ghana: a survey in an irrigation community. Trop Med Int Health 2004; 9(8):917-922.

49. Nanvya TN, Dakul DA, Mwansat GS. Schistosomiasis in Ndinjor district of Langtang north local government area of Plateau State, Nigeria. Nig J Parasitol 2011; 32(2):209-213.

50. Akinboye DO, Ajisebutu JU, Fawole O, Agbolade OM, Akinboye 00, Amosu AM, et al. Urinary schistosomiasis: Water contact frequency and infectivity among secondary school students in Ibadan, Nigeria. Nig J Parasitol 2011; 32(1):129-134.

51. Abdel-Wahab MF, Esmat G, Ramzy I, Narooz S, Medhat E, Ibrahim M, et al. The epidemiology of schistosomiasis in Egypt: Fayoum Governorate. Am J Trop Med Hyg 2000; 62(2):55-64.

52. Yameny AA. Validity of haematuria and proteinuria by chemical reagent strip for diagnosis of Schistosoma haematobium infection. J Biosci App Res 2017; 3(4):229-236. 